Objectives We used CMR with extracellular volume fraction (ECV) measurement to characterise cardiac involvement in relation to outcome in ATTR amyloidosis.

Methods Subjects comprised 263 patients with cardiac ATTR amyloidosis corroborated by grade $2-3{ }^{99} \mathrm{~m}$ Tc-DPD cardiac uptake, 17 with suspected cardiac ATTR amyloidosis (grade 1 ${ }^{99 \mathrm{~m}} \mathrm{Tc}-\mathrm{DPD}$ ) and 12 asymptomatic individuals with amyloidogenic transthyretin (TTR) mutations. Fifty patients with cardiac AL amyloidosis acted as disease controls.

Results In contrast to AL amyloidosis, asymmetric septal hypertrophy was present in 79\% of ATTR patients $(70 \%$ sigmoid septum and 30\% reverse septal curvature), whilst symmetric left ventricular hypertrophy (LVH) was present in only $18 \% ; 3 \%$ of patients has no LVH. In patients with cardiac amyloidosis, the pattern of LGE was always typical for amyloidosis (29\% subendocardial, 71\% transmural) including right ventricular LGE (96\%). 65 patients died during follow-up (19 \pm 14 months). ECV independently correlated with mortality and remained independent after adjustment for age, N-terminal pro-brain natriuretic peptide, ejection fraction, E/E' and left ventricular mass index (hazard ratio, 1.164; 95\% confidence interval, 1.066-1.271; $\mathrm{p}<0.01$ ).

Conclusions Asymmetric hypertrophy, traditionally associated with hypertrophic cardiomyopathy, is the commonest pattern of ventricular remodelling in ATTR amyloidosis. LGE imaging is typical in all patients with cardiac ATTR amyloidosis. ECV correlates with amyloid burden and provides incremental information on outcome even after adjustment for known prognostic factors.

\section{DETERMINANTS OF PULMONARY ARTERIAL REMODELLING IN COPD AND IMPLICATIONS FOR RIGHT VENTRICULAR REMODELLING}

Jonathan Weir-McCall, Patrick Liu-Shiu-Cheong, Allan D Struthers, Brian J Lipworth, J Graeme Houston. University of Dundee, UK

\subsection{6/heartjnl-2017-311399.25}

Introduction Pulmonary hypertension is a common complication of chronic obstructive pulmonary disease(COPD). Pulmonary arterial stiffening has been described in COPD both in those with pulmonary hypertension at rest and at exercise. Pulmonary pulse wave velocity (PWV) allows the non-invasive measurement of pulmonary arterial stiffening providing the potential for detection of early pulmonovascular changes before overt pulmonary hypertension sets in, but has not previously been assessed in COPD. The aim of the current study is thus to assess PWV in COPD and its effects on right ventricular(RV) remodelling.

Methods 58 participants with COPD underwent pulmonary function tests, six minute walk test, and cardiac MRI, while 21 age and sex matched healthy volunteers underwent cardiac MRI. 32 of the COPD patients underwent a follow-up MRI at 1 year to assess for longitudinal changes. Phase contrast imaging of the main pulmonary artery was performed in order to calculate pulmonary PWV using the flow-area technique.

Results Those with COPD demonstrated pulmonary arterial stiffening and pulmonary vascular remodelling with higher pulmonary artery area at end diastole (COPD: $2.36 \pm 0.56 \mathrm{~cm}^{2} /$ $\mathrm{m}^{1} .7$ vs. HC: $\left.2.14 \pm 0.28 \mathrm{~cm}^{2} / \mathrm{m}^{1} .7, \mathrm{p}=0.027\right)$, reduced pulsatility (COPD: $24.9 \pm 8.8 \%$ vs. HC: $30.6 \% \pm 11.3 \%, p=0.021$ ), reduced PAT (COPD: $104.0 \pm 22.9 \mathrm{~ms}$ vs. HC: $128.1 \pm 32.2$ $\mathrm{ms}, \mathrm{p}<0.001$ ) and higher PWV (COPD: $2.62 \pm 1.29 \mathrm{~ms}^{-1}$ vs. HC: $\left.1.78 \pm 0.72 \mathrm{~ms}^{-1}, \mathrm{p}=0.001\right)$. Pulmonary PWV was not associated with lung function, exercise capacity or right ventricular parameters. Cardiac remodelling in COPD was instead that of a reduced preload with a lower RV end diastolic volume (COPD: $53.6 \pm 11.1 \mathrm{ml}$ vs. HC: $59.9 \pm 13.0 \mathrm{ml}, \mathrm{p}=0.037$ ) and RV stroke volume (COPD: $31.9 \pm 6.9 \mathrm{ml} / \mathrm{m}^{2}$ vs. HC: 37.1 $\left.\pm 6.2 \mathrm{~m} / \mathrm{m}^{2} \mathrm{l}, \mathrm{p}=0.003\right)$. At follow-up those with the stiffest pulmonary arteries (top tertile of PWV) experienced no greater increase in RV mass than those with the lowest stiffness (bottom tertile of PWV), $p=0.29$ for difference.

Conclusion Pulmonary PWV is elevated in COPD, but does not have any significant association with right ventricular function or functional capacity. In fact remodelling in COPD is that of a reduced preload rather than the expected increased afterload.

\section{Comparison of Acceleration Algorithms in Whole-Heart Four}

\section{DIMENSIONAL FLOW CARDIOVASCULAR MAGNETIC RESONANCE: TWO-CENTRE, 1.5T, PHANTOM AND IN- VIVO VALIDATION STUDY}

${ }^{1}$ Pankaj Garg, ${ }^{2}$ Jos JM Westenberg, ${ }^{2}$ Pieter J van den Boogaard, ${ }^{1}$ Peter P Swoboda, ${ }^{1}$ Rahoz Aziz, ${ }^{1}$ James RJ Foley, ${ }^{1}$ Graham J Fent, ${ }^{2} \mathrm{FGJ}$ Tyl, ${ }^{2} \mathrm{~L}$ Coratella, ${ }^{2}$ Mohammed SM ElBaz, ${ }^{2}$ RJ van der Geest, ${ }^{3}$ David M Higgins, ${ }^{1} J o h n$ P Greenwood, ${ }^{1}$ Sven Plein. ${ }^{1}$ Leeds Institute of Cardiovascular and Metabolic Medicine (LICAMM), University of Leeds, Leeds, UK; ${ }^{2}$ Leiden University Medical Centre, Leiden, The Netherlands 3 Philips Healthcare, Guildford, UK

\subsection{6/heartinl-2017-311399.26}

Background Validation of four-dimensional (4D) flow CMR accelerated acquisition methods is needed to make them more robust for clinical applications. ${ }^{1}$ Our aim was to compare three widely-used acceleration methods in 4D flow CMR: 4D segmented fast-gradient-echo (4D- turbo-field-echo, 4D-TFE), 4D non-segmented gradient-echo with echo-planar imaging (4D- EPI) and 4D-k-t Broad-use Linear Acquisition Speed-up Technique accelerated TFE (4D- $k-t$ BLAST).

Methods CMR was performed in two institutions on identical $1.5 \mathrm{~T}$ systems. Acceleration methods were compared in static/

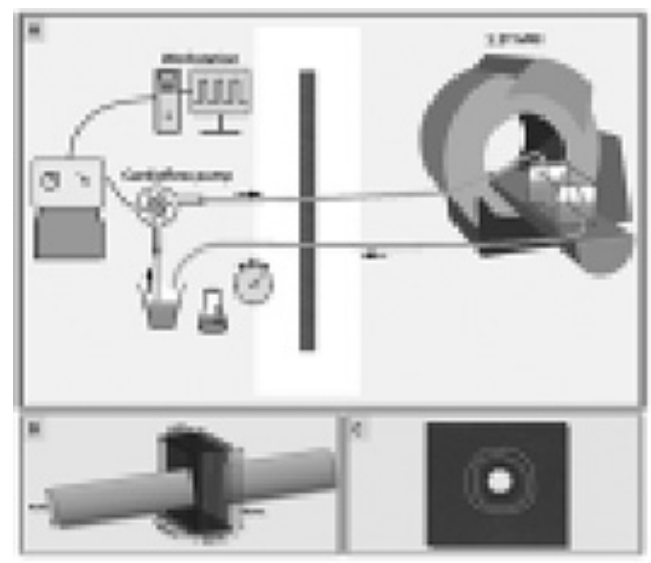

Abstract 026 Figure 1 Illustration to demonstrate static and pulsatile flow phantom setup. 

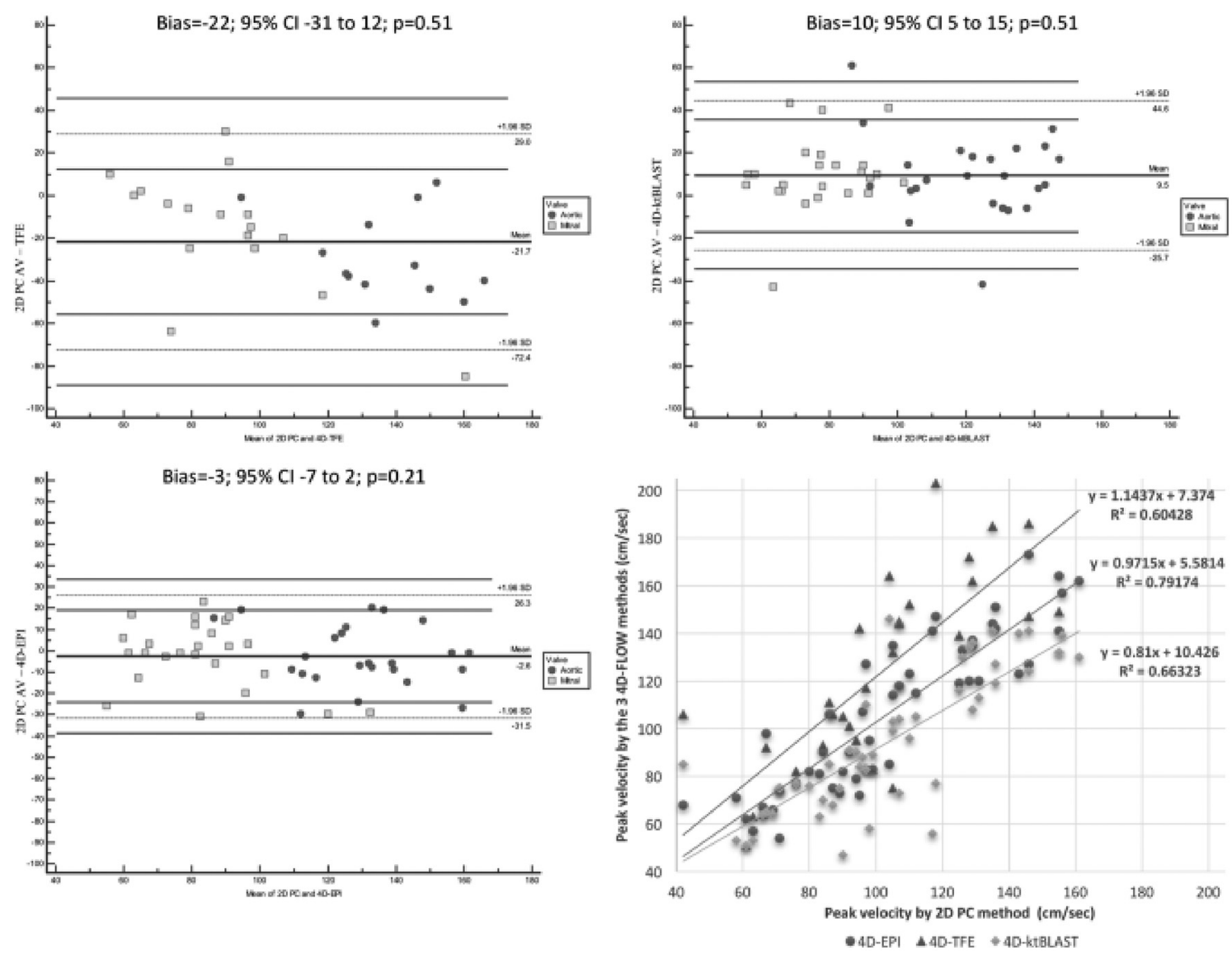

Abstract 026 Figure 2 Bland Altman analysis and scatter plots for the assessment of peak velocity using all the acceleration methods.

pulsatile phantoms (Figure 1) and 25 volunteers. In volunteers, the CMR protocol included: cines, $2 \mathrm{D}$ phase contrast $(\mathrm{PC})$ at the aortic valve (AV) and mitral valve (MV) and three wholeheart free-breathing (no respiratory motion correction) 4D flow CMR pulse sequences. Field-of-view, slices, phases (30), voxel size and VENC were the same for each subject. In volunteers, net acquisition time for each 4D flow sequence was recorded, as well as a visual grading of image quality on a four-point scale: 0 , no artefacts to 3 , non-evaluable.

Results For the pulsatile phantom experiments, the mean error against the reference flow by time beaker measurements for 4D-TFE was $4.9 \% \pm 1.3 \%$, for 4D-EPI $7.6 \% \pm 1.3 \%$ and for $4 \mathrm{D}-k-t$ BLAST $4.4 \% \pm 1.9 \%$. In vivo, acquisition time was shortest for $4 \mathrm{D}$-EPI at $7 \min 59 \mathrm{~s} \pm 2 \min 30 \mathrm{~s}$. 4D- EPI and 4D$k-t$ BLAST had minimal artefacts, while for 4D-TFE, $40 \%$ of $\mathrm{AV}$ and MV assessments were non-evaluable because of phase dispersion artefacts. Peak velocity assessment using 4D-EPI demonstrated best correlation to $2 \mathrm{D}$ PC (AV: $\mathrm{r}=0.78$, $\mathrm{p}<0.001 ; \mathrm{MV}: \mathrm{r}=0.71, \mathrm{p}<0.001)$. Coefficient of variability (CV) for net forward flow (NFF) volume was least for 4DEPI (7\%) (2D PC:11\%, 4D-TFE: 29\%, 4D-k-t BLAST: 30\%, respectively) (Figure 2, 3).

Conclusion Of the three 4D flow CMR methods tested, 4DEPI demonstrated the least susceptibility to artefacts, good image quality, modest agreement with the current reference standard for peak intra-cardiac velocities and the highest consistency of intra-cardiac flow quantifications.

Competing interests The authors declare that they have no competing interests.
Acknowledgement We thank Gavin Bainbridge, Caroline Richmond, Margaret Saysell and Petra Bijsterveld for their invaluable assistance in recruiting and collecting data for this study. Funding Sources This work was supported by the British Heart Foundation [FS/10/62/28409 to S.P.] and Dutch ZonMw [Project Number: 104003001 to J.W].

\section{REFERENCES}

1. Dyverfeldt $\mathrm{P}$, Bissell M, Barker AJ, Bolger AF, et al. 4D flow cardiovascular magnetic resonance consensus statement. J Cardiovasc Magn Reson 2015;17:72.

\section{T2 MAPPING IN ACUTE AND RECOVERED MYOCARDITIS: POTENTIAL ROLE IN CLINICAL SURVEILLANCE}

AS Lota, R Wassall, AD Scott, PD Gatehouse, R Wage, G Smith, U Tayal, BPHalliday, JS Ware, D Firmin, SA Cook, JG Cleland, DJ Pennell, SK Prasad.. Cardiovascular Biomedical Research Unit, Royal Brompton Hospital, London, UK

\subsection{6/heartjnl-2017-311399.27}

Background Acute myocarditis (AM) remains a challenging diagnosis with poorly defined markers of chronic active disease and/or progression to dilated cardiomyopathy. T2 mapping allows quantitative assessment of low- level myocardial oedema ${ }^{1}$ but requires further clinical evaluation alongside conventional biomarkers (troponin and BNP) prior to large-scale application. $^{2}$

Aim To prospectively evaluate the role of T2 mapping in the clinical surveillance of acute myocarditis. 